\title{
Kinematic reduction of reaction-diffusion fronts with multiplicative noise: Derivation of stochastic sharp-interface equations
}

\author{
A. Rocco, ${ }^{1,2}$ L. Ramírez-Piscina ${ }^{3}$ and J. Casademunt ${ }^{2}$ \\ ${ }^{1}$ CWI, Postbus 94079, 1090 GB Amsterdam, The Netherlands \\ ${ }^{2}$ Departament d'Estructura i Constituents de la Matèria, Facultat de Física, Universitat de Barcelona, Avinguda Diagonal 647, \\ E-08028 Barcelona, Spain \\ ${ }^{3}$ Departament de Física Aplicada, Universitat Politècnica de Catalunya, Avinguda Doctor Marañón 44, \\ E-08028 Barcelona, Spain \\ (Received 31 December 2001; published 17 May 2002)
}

\begin{abstract}
We study the dynamics of generic reaction-diffusion fronts, including pulses and chemical waves, in the presence of multiplicative noise. We discuss the connection between the reaction-diffusion Langevin-like field equations and the kinematic (eikonal) description in terms of a stochastic moving-boundary or sharp-interface approximation. We find that the effective noise is additive and we relate its strength to the noise parameters in the original field equations, to first order in noise strength, but including a partial resummation to all orders which captures the singular dependence on the microscopic cutoff associated with the spatial correlation of the noise. This dependence is essential for a quantitative and qualitative understanding of fluctuating fronts, affecting both scaling properties and nonuniversal quantities. Our results predict phenomena such as the shift of the transition point between the pushed and pulled regimes of front propagation, in terms of the noise parameters, and the corresponding transition to a non-Kardar-Parisi-Zhang universality class. We assess the quantitative validity of the results in several examples including equilibrium fluctuations and kinetic roughening. We also predict and observe a noise-induced pushed-pulled transition. The analytical predictions are successfully tested against rigorous results and show excellent agreement with numerical simulations of reaction-diffusion field equations with multiplicative noise.
\end{abstract}

DOI: 10.1103/PhysRevE.65.056116

PACS number(s): 82.40.Ck, 05.40.-a, 05.45.-a, 47.54. $+\mathrm{r}$

\section{INTRODUCTION}

The dynamics of localized solutions in the form of fronts or pulses in reaction-diffusion systems has received a great deal of attention for a long time in the context of nonequilibrium extended systems [1]. Examples of fronts formed by stable regions invading unstable or metastable ones are found in a large variety of physical, chemical, or biological systems, and have been studied in great detail [2-4]. In the context of excitable media and chemical waves, extended pulses do also exhibit a rich phenomenology [5-9]. As opposed to fronts, excitation waves or pulses are such that the region behind them eventually returns to the same linearly stable state that is ahead. In this case, in dimensions higher than one there may be open ends of the pulse region that give rise to spiral [two-dimensional (2D)] or scroll (3D) waves. There has been much interest in the study of such objects from the fundamental point of view of pattern forming dynamics, but also because of their potential applications in biological systems, such as in cardiac tissue [10]. In this paper we will not consider the case of open ends, so unless otherwise specified, we will refer to fronts and pulses without distinction under the common term of "fronts."

One aspect that has received increasing interest in recent years has been the effect of fluctuations of both internal and external origin on the dynamics and the roughening properties of fronts [11-19]. More recently, the effect of noise in pattern forming dynamics of chemical waves has been fostered by the development of the experimental capability of introducing external spatiotemporal noise in a controlled way in different photosensitive nonlinear chemical reactions, through the optical projection of computer-designed spatiotemporal fluctuations in the local illumination conditions, acting as a stochastic control parameter [20-23]. From a theoretical point of view, a common starting point to study fluctuations is in terms of master equations defining the evolution of reacting and diffusing particles in a lattice [11]. The connection of this microscopic level of description to the mesoscopic one in terms of Langevin field equations has proved a rather subtle issue, in connection with the distinction of the so-called pushed vs pulled fronts [4]. Only recently has a complete understanding of the instrinsically different nature of these two types of front, and the corresponding consequences concerning the presence of cutoffs [24-27] and the effects of fluctuations, started to emerge $[12,13,15-17]$. It has been shown, for instance, that pulled fronts define a universality class of kinetic roughening different from the Kardar-Parisi-Zhang (KPZ) universality class $[16,17]$. On the other hand, it has been shown that intrinsic noise at the microscopic level may induce a morphological instability at the macroscopic level of description [14]. In this paper we will be mostly concerned with the macroscopic description of pushed fronts with fluctuations, but also with how this description incorporates the transition to pulled fronts induced by the noise itself.

In the absence of noise, and in the appropriate limit, the description of fronts and pulses defined by reaction-diffusion field equations can be reduced by means of a moving boundary approximation to a kinematic description in terms of much simpler local equations $[9,28]$. This procedure, which is mathematically well grounded in the framework of the 
so-called inertial manifold reduction, can be carried out systematically, for instance, through asymptotic matching techniques using the front thickness as a small parameter defining a singular perturbation problem [29]. This is commonly used to relate macroscopic interface equations to orderparameter or phase-field descriptions in many problems involving interface dynamics, such as solidification [30,31], viscous fingering [32], etc. In the case of chemical pulses in excitable media in the limit of weak excitability, this leads to a local equation where the normal velocity of the pulse is a constant plus a correction proportional to curvature [9]. This is often called the eikonal equation. In the case of open ends, a similar local equation can be derived for the motion of the end point of the pulse $[9,28]$. For pushed fronts it can be shown that for smooth, long wavelength deformations of the front, the separation of time scales between the soft deformation modes and the internal degrees of freedom of the fields leads naturally to the same eikonal equation. For pulled fronts, however, this separation of time scales does not exist, the relaxation being algebraic instead of exponential, and a local moving-boundary approximation is not justified [29].

The kinematic description in terms of eikonal-like equations is a very useful approximation from both a theoretical and a practical point of view. In the context of the study of universality of fluctuation properties $[33,34]$ for instance, it leads naturally to the relevant effective universal description of a broad class of systems in terms of the KPZ equation [35]. It is also very useful for numerical simulation purposes, since it avoids resolving the fine structure of the front and the bulk degrees of freedom, which become irrelevant, dealing only with the kinematic degrees of freedom of an object of reduced dimension.

When noise is present in the original field equations, however, the situation is not so clear. Stochastic eikonal equations have proved useful in a phenomenological description of the dynamics of pulses and spiral waves in photosensitive chemical systems with external noise imposed on the illumination conditions $[21,22]$. Such a description, however, relied on some fitting parameters and some uncontrolled hypotheses on the way the noise must enter the kinematic equations. These results, together with the fact that the statistical properties of the noise present in those experiments are fully controlled, clearly call for a more "microscopic" derivation of stochastic kinematic equations corresponding to Langevin reaction-diffusion field equations with multiplicative noise, with no free parameters. The connection between bulk and interface fluctuations has been worked out so far only for equilibrium fluctuations in coarse-grained, Ginzburg-Landau-like equations [36-39]. In such cases, the sharp-interface limit can be taken at the level of the free energy, and the existence of a fluctuation-dissipation theorem then yields the proper way to incorporate thermal fluctuations into the effective interface equations. However, in many nonequilibrium systems, for instance in the context of reaction-diffusion problems, a free-energy or generically a Liapunov functional may not exist and no fluctuationdissipation relation may be invoked for external noise. In such cases the connection between the bulk description and the effective interface description in the presence of fluctuations must be worked out at the level of the dynamical equations.

The purpose of this paper is to address this point by deriving stochastic eikonal equations, including the complete specification of the noise statistics in connection with that of the noise in the mesoscopic field equations. In particular, we shall focus on the singular dependence on the spatial cutoff when noise is multiplicative, and its importance in the quantitative description of the statistical properties of the front fluctuations. The predictions will be tested against numerical simulations of reaction-diffusion equations, and also in cases where exact results are available concerning the spectrum of interface fluctuations. We will also see that the stochastic eikonal equation derived is consistent with the scenario of the pushed-pulled transition, and that changing the spatial cutoff or the noise intensity may have effects as drastic as changing the universality class of kinetic roughening of the front through that transition.

Although the method of obtaining the stochastic eikonal equation presented here is not a first-principles rigorous derivation, we will provide sufficient evidence to conclude that the result is the correct one to lowest order in noise intensity, including the singular dependence on the noise correlation cutoff (which involves a partial resummation to all orders), within the long time and length scale limits which are inherent to the kinematic description itself. However, it does not apply to situations in which the front dynamics is nonlocal, such as solidification fronts or viscous fingers, where a different type of formulation is appropriate even in the deterministic case $[30-32,40]$.

\section{KINEMATIC REDUCTION FOR GENERIC REACTION-DIFFUSION SYSTEMS}

Let us consider a vectorial field $\boldsymbol{\phi}(\mathbf{x}, t)$ with $N$ components $\boldsymbol{\phi}(\mathbf{x}) \equiv \phi_{1}(\mathbf{x}), \ldots, \phi_{N}(\mathbf{x})$ in a $d$-dimensional space with $\mathbf{x} \equiv x_{1}, \ldots, x_{d}$, which obeys a reaction-diffusion equation with multiplicative noise of the form

$$
\frac{\partial \boldsymbol{\phi}}{\partial t}=\hat{D} \nabla^{2} \boldsymbol{\phi}+\mathbf{f}(\boldsymbol{\phi})+\varepsilon^{1 / 2} \mathbf{g}(\boldsymbol{\phi}) \eta(\mathbf{x}, t),
$$

where $\eta(\mathbf{x}, t)$ is a Gaussian noise with zero mean and correlation given by

$$
\left\langle\eta(\mathbf{x}, t) \eta\left(\mathbf{x}^{\prime}, t^{\prime}\right)\right\rangle=2 \lambda^{-d} C\left(\left|\mathbf{x}-\mathbf{x}^{\prime}\right| / \lambda\right) \delta\left(t-t^{\prime}\right) .
$$

We take a one-component noise for simplicity, as the natural case when it originates in fluctuations of a single control parameter. The generalization of the formalism to multicomponent noise is straightforward. Notice the asymmetry with which we treat the spatial and temporal correlators of the noise. As we will see, this reflects a nontrivial issue related to the intrinsically different nature of the white noise limit in space as opposed to time. We have taken in Eq. (2) the noise as $\delta$-function correlated in time. This temporal white noise limit is well behaved, once a prescription for the multiplicative noise term in Eq. (1) has been chosen. For external fluctuations the physically relevant prescription is to consider the 
white noise as the limit of some properly defined correlated noise. This corresponds to the well-known Stratonovich prescription [41]. On the other hand, the spatial noise must always be defined as colored, its white (uncorrelated) limit being intrinsically ill defined. Hence the notation with the function $\lambda^{-d} C(r / \lambda)$ in the correlator, meaning a general correlation function, dependent on some correlation length $\lambda$, which in the limit $\lambda \rightarrow 0$ is such that $\lambda^{-d} C(r / \lambda) \rightarrow \delta(r)$. The fact that the spatial continuum limit $\lambda \rightarrow 0$ does not exist in the Stratonovich interpretation is thus reflected in the fact that, even if $\lambda$ is much smaller than any other length scale in the problem, the existence of such a microscopic cutoff always shows up in the quantitative description of the large scale behavior and cannot be reabsorbed in a redefinition of parameters. That is, the noise cannot be considered as effectively white in space, regardless of how small the noise correlation length is.

The multiplicative noise term present in Eq. (1) has an average value different from zero. Applying the Novikov theorem [42], we get

$$
\varepsilon^{1 / 2}\langle\mathbf{g}(\boldsymbol{\phi}) \eta(\mathbf{x}, t)\rangle=\varepsilon \lambda^{-d} C(0)\langle\mathbf{G}(\boldsymbol{\phi})\rangle,
$$

where

$$
G_{i}(\boldsymbol{\phi}) \equiv \sum_{j} \frac{\partial g_{i}(\boldsymbol{\phi})}{\partial \phi_{j}} g_{j}(\boldsymbol{\phi}) .
$$

This suggests separating the average contribution from the multiplicative noise term and rewriting Eq. (1) in terms of a renormalized potential and a zero average noise,

$$
\frac{\partial \boldsymbol{\phi}}{\partial t}=\hat{D} \nabla^{2} \boldsymbol{\phi}+\mathbf{h}(\boldsymbol{\phi})+\varepsilon^{1 / 2} \boldsymbol{\Omega}(\boldsymbol{\phi}, \mathbf{x}, t),
$$

where

$$
\mathbf{h}(\boldsymbol{\phi})=\mathbf{f}(\boldsymbol{\phi})+\varepsilon \lambda^{-d} C(0) \mathbf{G}(\boldsymbol{\phi})
$$

and

$$
\boldsymbol{\Omega}(\boldsymbol{\phi}, \mathbf{x}, t)=\mathbf{G}(\boldsymbol{\phi}) \eta(\mathbf{x}, t)-\varepsilon^{1 / 2} \lambda^{-d} C(0) \mathbf{G}(\boldsymbol{\phi}) .
$$

Here the new noise $\boldsymbol{\Omega}$ has zero average. Notice that this decomposition corresponds to transforming Eq. (1) into its equivalent Itô stochastic equation in the white noise limit. Accordingly, the stochastic term $\Omega$ reduces to

$$
\lim _{\lambda \rightarrow 0} \boldsymbol{\Omega}(\boldsymbol{\phi}, \mathbf{x}, t)=\mathbf{G}(\boldsymbol{\phi}) \eta_{I}(\mathbf{x}, t),
$$

where $\eta_{I}(\mathbf{x}, t)$ is now a white noise in the Itô interpretation. The deterministic term $\mathbf{h}$ thus includes noise effects through the so-called Stratonovich term that has been added to $\mathbf{f}$. These noise effects on the deterministic part of Eq. (5) depend on a "dressed" noise intensity $\varepsilon_{\lambda}$ which contains the singular dependence on the spatial cutoff in the form of the "bare" $\varepsilon$ as $\varepsilon_{\lambda} \equiv \varepsilon C(0) \lambda^{-d}$. An important point is that the spatial white noise limit in the continuum equations can only be mathematically well defined for an Itô noise. This has been proved rigorously for relatively broad classes of equa- tions (see, for instance, Refs. [43,44]) in one dimension. On the contrary, this cannot be the case for a Stratonovich noise, as is obvious from the singular dependence on $\lambda$. The practical implications of these facts are that, while the singular contribution of $\lambda$ in the Stratonovich term must be kept explicitly, the dependence on $\lambda$ contained in $\boldsymbol{\Omega}$ may indeed be weak (nonsingular), and negligible if $\lambda$ is much smaller than the other length scales in the problem, in particular the front thickness. This is rigorous only in one dimension, for instance in the case discussed in Ref. [15]. In higher dimensions, a residual strong dependence of a given quantity on the cutoff $\lambda$ associated with the noise term $\boldsymbol{\Omega}$ can only be ruled out empirically, as we check in our numerical simulation in the sections below. Therefore, the splitting of the Stratonovich noise by means of a deterministic term plus an Itô multiplicative noise has the virtue of isolating the singular dependence on the microscopic cutoff. This will also define a useful partial resummation of orders in $\varepsilon_{\lambda}$. With the above considerations, the theory here presented is thus expected to be correct to first order in $\varepsilon$, to all orders in $\varepsilon / \lambda$, and to order $\lambda^{0}$ for the regular dependence of $\boldsymbol{\Omega}$ on the noise correlation length.

Equation (5) will be our starting point. We are interested in representing the dynamics of a front obeying Eq. (5) as the evolution of a $(d-1)$-dimensional surface. In this effective dynamics we assume the details of the front structure (at scales of the order of or smaller than the front thickness) to be unimportant. In what follows we will consider the evolution of a one-dimensional front embedded in a twodimensional system. In the procedure we are going to apply we will write the evolution equation (5) in the curvilinear coordinates defined by the 1D front in the sharp-interface limit, and obtain the evolution equation for this front as a solvability problem with the basic assumption that curvature and noise are small perturbations.

Before proceeding with the formal derivation, let us first point out some subtleties related to the stochastic case as opposed to the deterministic one. In the latter, it is customary to define a curvilinear coordinate system $(s, r)$ in which $r$ $=0$ stands for the curve representing the front position, which can be associated, for instance, with a level curve of the appropriate field. The scheme assumes that the front thickness is small compared to the radius of curvature, and that the relaxation of the internal degrees of freedom of the front is fast compared to the time scale of the long wavelength deformations of the front. When noise is present in the field equation, the appropriate curvilinear coordinate system cannot be associated with level curves, which are very rough at length scales smaller than the front thickness. On the other hand, at larger scales, which are the ones we are interested in, a coarse-grained description makes perfect sense. This is actually implicit in the very idea of the stochastic eikonal equation. One can think of different schemes to explicitly define such a coarse-grained description, all of them equivalent. However, since the rest of the derivation cannot be carried out explicitly in full rigor in any of those, and since the result is expected to be independent of the details of the definition of the coarse graining, we will proceed more or less heuristically. In essence this is a reformulation of the 
approach introduced for the derivation of the diffusive wandering of fronts in one dimension discussed in Ref. [13]. There, the basic idea was that only the low-frequency components of the noise are responsible for the front wandering so the high-frequency components can be implicitly integrated out. The effect of the high-frequency components of the noise is thus to renormalize the mean front profile. As a consequence, they renormalize the front velocity, and also the diffusion coefficient. More precisely, this means in our case that the high-frequency renormalization is carried out by the Stratonovich term in the function $\mathbf{h}$. Once this term is explicitly extracted, the remainder is an Ito multiplicative noise. Then the high-frequency components of this term are irrelevant and can be averaged out, while the low-frequency components will be responsible for the roughening of the front at scales larger than the coarse-graining length. In the case of 1D fronts, the resulting diffusion coefficient for the front wandering was rederived rigorously in Ref. [15]. Unfortunately, the identification of the collective variable in 1D cannot be immediately generalized to higher dimensions, so we must still rely on a less precise formulation and check the consistency with rigorous results and numerical simulations a posteriori.

After the above considerations, we can make the following theoretical construction. We assume we have solved the field equations with noise without any approximation. We now coarse-grain the fields with some local average procedure, in both time and space, and use the coarse-grained fields to define a curvilinear coordinate system based, for instance, in terms of level curves at any time. At short length and time scales this coordinate system is smooth and, in principle, we could write the full field equation (still with the bare fields) in these curvilinear coordinates. In the absence of noise, an expansion in the front thickness would unambiguously yield the terms that are dominant in the range that the eikonal equation is devised for, namely, for sufficiently long length scales (small curvatures) and long time scales. Terms such as second derivatives in $s$ and the time derivative would automatically drop out of the description. In the presence of stochastic noise, this is not so automatic unless the fields themselves are coarse grained so that they are also sufficiently smooth in space and time. We assume that, for the coarse-grained fields, the order of the different terms in the front thickness will be the same as for the deterministic case, when such an expansion makes sense (excluding pulled fronts, for instance). We claim that this assumption is the one implicit in the very idea of the existence of a stochastic kinematic formulation of the front dynamics. Then, for the coarse-grained fields, Eq. (5) is expected to reduce, in analogy to the deterministic case, to

$$
\begin{gathered}
\hat{D} \frac{\partial^{2} \boldsymbol{\phi}}{\partial r^{2}}+\hat{D} \kappa(s, t) \frac{\partial \boldsymbol{\phi}}{\partial r}+\mathbf{h}(\boldsymbol{\phi})+v_{n}(s, t) \frac{\partial \boldsymbol{\phi}}{\partial r} \\
+\varepsilon^{1 / 2} \boldsymbol{\Omega}(\boldsymbol{\phi} ; r, s ; t)=0,
\end{gathered}
$$

where $\kappa$ is the local curvature and $v_{n}$ the normal velocity of the front. This normal velocity provides the evolution of the curvilinear coordinates in which Eq. (5) takes the form of
Eq. (9), and is the fundamental quantity we are interested in. The noise term in Eq. (9) must also be considered as coarse grained, with the high-frequency high-wave-number components integrated out.

At this point of the derivation it is useful to consider the 1D problem which corresponds to the zeroth order of the eikonal description. This is defined by neglecting the curvature and the fluctuating term in Eq. (9):

$$
0=\hat{D} \frac{\partial^{2} \boldsymbol{\phi}_{0}}{\partial r^{2}}+\bar{v}\left(\varepsilon_{\lambda}\right) \frac{\partial \boldsymbol{\phi}_{0}}{\partial r}+\mathbf{h}\left(\boldsymbol{\phi}_{0}\right) .
$$

This is the eigenvalue problem that gives the renormalized velocity $\bar{v}$ in the $1 \mathrm{D}$ problem as obtained in Refs. [12,13]. Note that this equation does contain noise effects through the high-frequency renormalization provided by the Stratonovich term. In fact, the effective velocity $\bar{v}\left(\varepsilon_{\lambda}\right)$ resulting from Eq. (10) has contributions from all orders in the dressed noise intensity $\varepsilon_{\lambda}$. An explicitly first order in $\varepsilon_{\lambda}$ approximation will be given in the Appendix. In the problem defined by Eq. (9) the curvature and fluctuations will be taken as small perturbations. Hence we assume for the field $\boldsymbol{\phi}$ and the velocity $v_{n}$ expansions of the form

$$
\begin{gathered}
\boldsymbol{\phi}(r, s, t)=\boldsymbol{\phi}_{0}(r)+\delta \boldsymbol{\phi}(r, s, t), \\
v_{n}(s, t)=\bar{v}\left(\varepsilon_{\lambda}\right)+\beta\left(\varepsilon_{\lambda}\right) \kappa(s, t)+\delta v(s, t),
\end{gathered}
$$

where $\boldsymbol{\phi}_{0}(\boldsymbol{r})$ and $\bar{v}\left(\varepsilon_{\lambda}\right)$ are the solution of the 1D problem of Eq. (10). The term $\beta \kappa(s, t)$ is a curvature correction and $\delta v(s, t)$ describes fluctuations. Linearizing in both perturbations, $\delta \boldsymbol{\phi}(r, s)$ then satisfies

$$
\begin{aligned}
0= & \hat{\Gamma} \delta \boldsymbol{\phi}+(\beta \kappa+\delta v) \frac{\partial \boldsymbol{\phi}_{0}}{\partial r}+\hat{D} \kappa \frac{\partial \boldsymbol{\phi}_{0}}{\partial r} \\
& +\varepsilon^{1 / 2} \boldsymbol{\Omega}\left(\boldsymbol{\phi}_{0} ; r, s ; t\right),
\end{aligned}
$$

where

$$
\hat{\Gamma}=\hat{D} \frac{\partial^{2}}{\partial r^{2}}+\bar{v}\left(\varepsilon_{\lambda}\right) \frac{\partial}{\partial r}+\left.\frac{\partial \mathbf{h}}{\partial \boldsymbol{\phi}}\right|_{\boldsymbol{\phi}=\boldsymbol{\phi}_{0}} .
$$

Taking the derivative of Eq. (10) with respect to $r$, it is a simple matter to prove that

$$
\mathbf{u}_{0}=\frac{\partial \boldsymbol{\phi}_{0}}{\partial r}
$$

is the right eigenvector of $\hat{\Gamma}$ with zero eigenvalue. Due to the non-Hermiticity of $\hat{\Gamma}$, finding an analytic expression for the left eigenvector $\mathbf{u}^{0}$ is not trivial. Notice that, because of the vectorial character of the field, the simple expression obtained in Ref. [13] for scalar fields is in general not applicable. Nevertheless, the corresponding eigenvector can always be obtained at least numerically.

Now, taking Eq. (13) and performing the scalar product with $\mathbf{u}^{0}$, we obtain 


$$
\begin{aligned}
& \kappa\left(\mathbf{u}^{0}, \hat{D} \mathbf{u}_{0}\right)+\beta \kappa\left(\mathbf{u}^{0}, \mathbf{u}_{0}\right)+\varepsilon^{1 / 2}\left(\mathbf{u}^{0}, \mathbf{\Omega}\left(\boldsymbol{\phi}_{0} ; r, s ; t\right)\right) \\
& \quad+\delta v(s, t)\left(\mathbf{u}^{0}, \mathbf{u}_{0}\right)=0
\end{aligned}
$$

where the scalar product is defined by

$$
(\mathbf{f}, \mathbf{g})=\sum_{i} \int d r f_{i}(r) g_{i}(r) .
$$

Owing to the independence of the two first-order perturbations (curvature and fluctuations), we get

$$
\beta\left(\varepsilon_{\lambda}\right)=-\frac{\left(\mathbf{u}^{0}, \hat{D} \mathbf{u}_{0}\right)}{\left(\mathbf{u}^{0}, \mathbf{u}_{0}\right)}
$$

and

$$
\delta v(s, t)=-\varepsilon^{1 / 2} \frac{\left(\mathbf{u}^{0}, \mathbf{\Omega}\left(\boldsymbol{\phi}_{0} ; r, s ; t\right)\right)}{\left(\mathbf{u}^{0}, \mathbf{u}_{0}\right)} .
$$

The stochastic process (19) is not white since the highfrequency components of $\boldsymbol{\Omega}$ have been integrated out by the coarse-graining procedure. However, we can now restore them harmlessly by replacing $\boldsymbol{\Omega}$ with the original multiplicative white noise process. By doing this, we are modifying the part that is not intended to be accounted for by the eikonal description, while the treatment becomes simpler. Analogously, once the dependence on the cutoff $\lambda$ has been explicitly worked out, we can take the process (19) as $\delta$-function correlated in space. We will explicitly check the limit of validity of the eikonal description here proposed when scales comparable to the front thickness are reached, in the sections below.

The resulting stochastic eikonal equation with the explicit dependence on the original noise parameters then takes the form

$$
v_{n}(s, t)=\bar{v}\left(\varepsilon_{\lambda}\right)+\beta\left(\varepsilon_{\lambda}\right) \kappa(s, t)+D_{f}^{1 / 2}\left(\varepsilon, \varepsilon_{\lambda}\right) \zeta(s, t),
$$

where $\bar{v}\left(\varepsilon_{\lambda}\right)$ is defined by Eq. (10), $\beta\left(\varepsilon_{\lambda}\right)$ is given by Eq. (18), and the noise $\zeta(s, t)$ is a zero mean Gaussian white process with

$$
\left\langle\zeta(s, t) \zeta\left(s^{\prime}, t^{\prime}\right)\right\rangle=2 \delta\left(s-s^{\prime}\right) \delta\left(t-t^{\prime}\right),
$$

which follows from the statistical properties of $\Omega$ with

$$
D_{f}\left(\varepsilon, \varepsilon_{\lambda}\right)=\varepsilon \frac{\int d r \sum_{i, j} u_{i}^{0} u_{j(0)} g_{i}\left(\boldsymbol{\phi}_{0}\right) g_{j}\left(\boldsymbol{\phi}_{0}\right)}{\left(\mathbf{u}^{0}, \mathbf{u}_{0}\right)^{2}} .
$$

Note that the dependence of $D_{f}$ on the dressed noise intensity $\varepsilon_{\lambda}$ comes from the dependence on the same quantity of $\boldsymbol{\phi}_{0}$ (and hence of $\mathbf{u}_{0}$ and $\mathbf{u}^{0}$ ) as the solution of the renormalized problem of Eq. (10).

The above equations constitute the main result of the first part of the paper. Although the derivation is not rigorous because the coarse graining could not be carried out explic- itly, the result is appealing from the theoretical point of view in that it separates the problem into an effective deterministic one, where the original field equations are modified by additional deterministic terms which depend on noise parameters, plus an additive noise that would be the necessary one to describe the wandering of the problem in one dimension. For the renormalized one-dimensional deterministic problem, therefore, the two present perturbations, curvature and noise, decouple from each other.

An important point to emphasize here is the separate dependence of the result on two noise parameters, namely, $\varepsilon$ and $\varepsilon_{\lambda}$. While the renormalized velocity and the coefficient of the curvature depend solely on $\varepsilon_{\lambda}$, the effective noise intensity $D_{f}$ depends separately on both. This clearly illustrates how the ultraviolet cutoff is an additional parameter of the problem when the noise is multiplicative, corresponding to the fact that the continuum limit is not well defined for noise $\delta$-function correlated in space. It is also important to remark that our derivation procedure is expected to be valid for small noise intensity $\varepsilon$, but contains all orders in $\varepsilon_{\lambda}$. This was already the case in the one-dimensional derivation of Ref. [13], where the small noise approximation was phrased in terms of a separation of time scales. The connection between that scale separation, the coarse-graining procedure, and the small noise expansion was recently clarified in Ref. [18], where a rigorous derivation of the result of Ref. [13] was presented for the case of a single-component field in one dimension, in terms of suitable projection techniques. Unfortunately, that rigorous derivation is based on the identification of a specific collective coordinate, which has no simple extension to higher dimensions. Nevertheless, the fact that this approximate procedure has proved correct in 1D gives further support to our main result above, which is not claimed to be rigorously proved. In the following sections we will check this prediction against analytical results and numerical simulations of the full reaction-diffusion equations in explicit examples. We will see that the dependence on the cutoff $\lambda$ is essential not only for a quantitative description of the problem, but also to predict nontrivial phenomena such as the transition to pulled fronts, in which the whole eikonal description fails. This failure of the present description is signaled by the vanishing of the effective noise intensity $D_{f}$ when that point is reached. In fact, $D_{f}$ is linear in $\varepsilon$ to lowest order, but has a complicated dependence on $\varepsilon_{\lambda}$. As we will explicitly see, the partial resummation of orders in $\varepsilon_{\lambda}$ captures important physical features of the problem. For instance, it allows the nonmonotonic dependence and eventual vanishing of the front diffusion coefficient $D_{f}$. We expect the pushed-pulled transition to occur exactly at this point. Another qualitative change captured by the above resummation is the destruction of the front itself, associated with the fact that the front thickness may diverge in some circumstances. This phenomenon is signaled by a divergence of $D_{f}$ at some finite value of $\varepsilon_{\lambda}$.

Although the predictions above are expected to be correct for $\epsilon_{\lambda} \sim 1$ as long as $\epsilon \ll 1$, in practice this may be limited by the fact that $\bar{v}\left(\varepsilon_{\lambda}\right)$ and the Goldstone modes are not in general analytically known. In this case one can rely on numeri- 


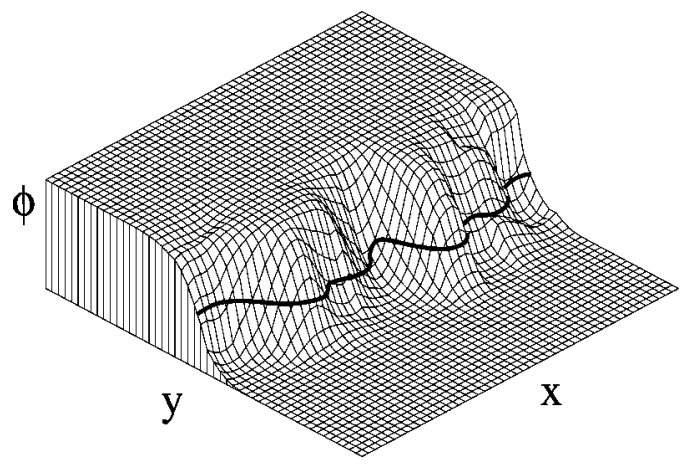

FIG. 1. An example of a noisy front with a level curve that defines the precise location of the front.

cal resolutions of the eigenvalue problem posed by Eq. (10), or alternatively one can find $\bar{v}\left(\varepsilon_{\lambda}\right)$ as a systematic expansion in powers of $\varepsilon_{\lambda}$ as described in the Appendix. On the other hand, it is worth remarking that $\beta$ can be a nontrivial function of $\varepsilon_{\lambda}$ only for multicomponent fields, that is, for pulses. For one-component fields (fronts) the coefficient $\beta$ is not renormalized by noise.

We remark that this derivation is meaningful only when pulses are involved or, in the case of fronts, when the relevant dynamical regime is the pushed one. In Sec. V of this paper, we shall give more details about the main differences between pushed and pulled fronts, and about their different responses to noise. For the time being, we point out that the lack of time-scale separation between the relaxation of the zero mode and the other eigenmodes of the spectral operator for pulled fronts prevents us in general from constructing a local equation for the interface motion [29]. Examples of reaction-diffusion systems that do not admit local kinematic descriptions are the phase-field formulations of solidification $[30,40]$ or viscous fingering [32]. In those cases a nonlocal interface equation does exist so an extension of our derivation is in principle feasible. This may be particularly interesting in cases such as those in Refs. [40,32] where the relevant fluctuations may be external.

\section{KINETIC ROUGHENING AND CONNECTION TO EQUILIBRIUM FLUCTUATIONS}

In the previous section, we derived a stochastic sharp interface approximation for a generic reaction-diffusion (RD) system with multiplicative noise. A pictorial description of a noisy front is given in Fig. 1. We see in this figure that noise in the RD system induces fluctuations in the front shape, thus generating roughening of the sharp interface that should emerge at the eikonal level of description. The identification of universality classes of kinetic roughening will come naturally at this level of description. In particular, we will establish the connection with the universality classes defined by the Kardar-Parisi-Zhang equation [35] and the EdwardsWilkinson (EW) equation [45].

The stochastic eikonal equation (20) is written in intrinsic, rotation invariant form. For the purposes of scaling theory it is convenient to write it in Cartesian coordinates. The front location is then given as $y=h(x, t)$. Retaining only the rel- evant nonlinear terms in the renormalization group (RG) sense, we then recover the KPZ equation

$$
\frac{\partial h}{\partial t}=\nu \frac{\partial^{2} h}{\partial x^{2}}+\frac{\lambda}{2}\left(\frac{\partial h}{\partial x}\right)^{2}+\mu(x, t)
$$

with

$$
\left\langle\mu(x, t) \mu\left(x^{\prime}, t^{\prime}\right)\right\rangle=2 D_{\mathrm{KPZ}} \delta\left(x-x^{\prime}\right) \delta\left(t-t^{\prime}\right) .
$$

The KPZ parameters turn out to be related to the eikonal ones as

$$
D_{\mathrm{KPZ}}=D_{f}, \quad \nu=\beta, \quad \lambda=\bar{v} .
$$

In the special case of $\bar{v}=0$, the EW equation is obtained,

$$
\frac{\partial h}{\partial t}=\nu \frac{\partial^{2} h}{\partial x^{2}}+\mu(x, t)
$$

with

$$
\left\langle\mu(x, t) \mu\left(x^{\prime}, t^{\prime}\right)\right\rangle=2 D_{\mathrm{EW}} \delta\left(x-x^{\prime}\right) \delta\left(t-t^{\prime}\right),
$$

and again

$$
D_{\mathrm{EW}}=D_{f}, \quad \nu=\beta .
$$

These equations are well known to be the paradigm for many different growth processes [33,34]. Even if the microscopic dynamics of the system under study may correspond to different equations of motion for the respective interfaces or surfaces, nevertheless the KPZ and EW equations do capture the universal features of the system, namely, the scaling properties of fluctuations.

Usually, such effective equations cannot be derived from the original microscopic description of the particular systems and are introduced on a phenomenological basis, relying on the claim of universality within a RG framework. Nonuniversal quantities such as prefactors of scaling functions, affected, for instance, by the noise intensity in the interface equation, cannot be derived. In our case, we are able to compute the noise intensity in the eikonal equation so we can also predict the nonuniversal prefactors if the noise is known at the reaction-diffusion level of description. For instance, not only can the scaling of the interface roughness with system size be predicted, but also the actual values of average interface roughness in terms of the original microscopic parameters of the RD model are worked out.

As a test of our derivation we will now check consistency with equilibrium fluctuation theory. The connection between bulk thermal fluctuations and fluctuations of the interface between thermodynamical phases can be established rigorously in the case of equilibrium fluctuations. This is possible because a free-energy functional does exist and the sharpinterface limit can be performed at the level of the free energy itself. Then, the fluctuations can be obtained independently from the free energy at each level of description (either bulk or interface fluctuations), consistently with the fluctuation-dissipation theorem. The important difference 
here is that no dynamical equation must be invoked, but only equilibrium properties. On the contrary, in the more general case where there is no fluctuation-dissipation theorem and not even a free-energy functional, we must rely on dynamical equations. In the case of equilibrium fluctuations, however, we must reproduce the known correct result. As we will see below, this case falls in the EW universality class.

The general solution of the EW equation, Eq. (26), is known [34]. Consider the interface $h(x, t)$ and its discrete Fourier transform $\hat{h}_{q}(t)$, defined through

$$
h(x, t)=\sum_{q} \hat{h}_{q}(t) \exp (\text { iqx }) .
$$

It is possible to show [34] that the long time limit of the spectrum $S(q, t)=\left\langle\left|\hat{h}_{q}(t)\right|^{2}\right\rangle$ is given by the expression

$$
\lim _{t \rightarrow \infty} S(q, t)=\frac{1}{L} \frac{D_{\mathrm{EW}}}{\nu q^{2}} .
$$

Our strategy now will be to calculate explicitly the spectrum (30) in terms of the coefficients predicted for our eikonal equation, and then show that the resulting expression coincides with the independent result that can be obtained from equilibrium fluctuation theory. Hence we insert an additive noise in the original RD system, that is, in setting $g=1$,

$$
\frac{\partial \phi}{\partial t}=D \nabla^{2} \phi+F(\phi)+\varepsilon^{1 / 2} \eta(\mathbf{x}, t),
$$

with

$$
\left\langle\eta(\mathbf{x}, t) \eta\left(\mathbf{x}^{\prime}, t^{\prime}\right)\right\rangle=2 \delta\left(\mathbf{x}-\mathbf{x}^{\prime}\right) \delta\left(t-t^{\prime}\right) .
$$

We consider an $F(\phi)$ with a symmetric double-well form, i.e., the deterministic part of Eq. (31) has a 1D solution with zero velocity $\phi=\phi_{0}(x)$. This corresponds to the usual timedependent Gizburg-Landau Langevin equation for a nonconserved order parameter (model A in the Hohenberg-Halperin classification [36]), where noise intensity must be identified as $\varepsilon=k_{B} T$. Since $g=1$, our expression for the noise intensity at the sharp interface level takes the simpler form

$$
D_{f}=\frac{\varepsilon}{\int_{-\infty}^{\infty} d x\left(\partial \phi_{0} / \partial x\right)^{2}},
$$

which, according to Eq. (30) and performing the identifications of Eq. (28) with $\beta=D$, produces

$$
\lim _{t \rightarrow \infty}\left\langle\left|\hat{h}_{q}(t)\right|^{2}\right\rangle=\frac{\varepsilon}{L D \int_{-\infty}^{\infty} d x\left(\partial \phi_{0} / \partial x\right)^{2}} \frac{1}{q^{2}} .
$$

On the other hand, we can take the sharp-interface limit of the free Ginzburg-Landau free energy. The calculation is standard (see, for instance, Ref. [37]) and yields the interface free energy

$$
F_{I}=\sigma \int d x \sqrt{1+\left(\frac{\partial h}{\partial x}\right)^{2}}
$$

where the parameter $\sigma$ is identified as the interfacial tension and can be evaluated from the bulk free energy of the system [37] as

$$
\sigma=D \int d x\left(\frac{\partial \phi_{0}}{\partial x}\right)^{2}
$$

where $\phi_{0}$ is the corresponding kink solution. For soft (long wavelength) deformations of the interface, the excess free energy reads

$$
\Delta F_{I} \approx \frac{\sigma}{2} \int d x\left(\frac{\partial h}{\partial x}\right)^{2}
$$

and the corresponding stationary spectrum of fluctuations, consistent with the fluctuation-dissipation theorem, takes the form [37]

$$
\left\langle\left|\hat{h}_{q}\right|^{2}\right\rangle=\frac{k_{B} T}{L \sigma q^{2}}
$$

Using Eq. (36), this expression yields the same result as Eq. (34). This proves that the front roughening obtained from our derivation is exact in the case of equilibrium fluctuations, and by extension in the additive noise case. We have shown this for the case of a nonconserved order parameter, for which the connection between the microscopic (Ising-like) and sharp-interface levels of description is indeed well established. In the conserved case (model B of Ref. [36]), the projection to a sharp-interface description yields a nonlocal equation and therefore lies outside the validity of our theory. Similarly, equilibrium fluctuations have also been studied in both sharp-interface [38] and phase-field $[39,40]$ formulation in the context of solidification, which also yields nonlocal interface dynamics. The universality classes in those cases are not well established.

\section{APPLICATION TO A PROTOTYPE MODEL OF FRONT PROPAGATION}

To illustrate our general theory we consider here as an example the propagation of a scalar front

$$
\frac{\partial \phi}{\partial t}=D \nabla^{2} \phi+F(\phi, a)+\varepsilon^{1 / 2} g(\phi) \eta,
$$

with the noise correlator defined as in Eq. (2). We specify our prototype model through the following definitions:

$$
\begin{gathered}
F(\phi)=\phi(1-\phi)(\phi+a), \\
g(\phi)=\phi(1-\phi),
\end{gathered}
$$

and we will consider a front of the $\phi=1$ state invading the $\phi=0$ one. The constant $a$ is a control parameter. As is well known for fronts without fluctuations, the deterministic force given by Eq. (40) leads to different modes of front propaga- 
tion depending on the value of $a$ [2]. The front velocity also depends on $a$. The choice of the coupling function (41) for the multiplicative noise term of Eq. (39) is the simplest one that preserves the stationary states $\phi=0$ and $\phi=1$. The fact that the noise term vanishes in the two asymptotic states prevents nucleation phenomena in the invaded state. The form of $g(\phi)$ is also such that the multiplicative noise term arises naturally as the fluctuation of the control parameter $a$. Moreover, for the prototype model here proposed, the corresponding function $h(\phi)$ defined in Eq. (6) which appears in the renormalized equation takes the same functional form as $f(\phi)$, only with renormalized coefficients. This allows a simpler analytical treatment and intepretation of the results.

\section{A. The 1D case revisited}

This prototype model has already been analyzed for 1D in Refs. [12,13], where in the regime $-\varepsilon_{\lambda}<a<1 / 2-\varepsilon_{\lambda}$ it is shown to result in a renormalized average velocity

$$
\bar{v}=\frac{2 a+1}{\sqrt{2\left(1-2 \varepsilon_{\lambda}\right)}}
$$

and in the diffusion coefficient

$$
D_{f}=\varepsilon \frac{\int_{-\infty}^{\infty} d \xi e^{2 \bar{v} \xi}\left(d \phi_{0} / d \xi\right)^{2} g^{2}\left(\phi_{0}\right)}{\left[\int_{-\infty}^{\infty} d \xi e^{\bar{v} \xi}\left(d \phi_{0} / d \xi\right)^{2}\right]^{2}}
$$

Here $\phi_{0}$ is the solution of the zeroth-order equation:

$$
\frac{d^{2} \phi_{0}}{d \xi^{2}}+\bar{v} \frac{d \phi_{0}}{d \xi}+h\left(\phi_{0}\right)=0
$$

Notice the dependence of both these quantities on $\lambda$ through the $\varepsilon_{\lambda}$ parameter. Specifically about the diffusion coefficient, the first order dependence in $\varepsilon$ is apparent, while the functions present in the integral and defined through Eq. (44) contain all orders in $\varepsilon_{\lambda}$. This is in contrast to the renormalized velocity, which depends solely on $\varepsilon_{\lambda}$. This means that both $\varepsilon$ and $\lambda$ can be determined independently from separate measurements of both the ballistic and the diffusive components of the front propagation. This is quite remarkable since it provides indirect means of measurement of the (microscopic) noise, which may not be directly accessible in many cases.

As an illustrative and well-controlled example, we have explicitly tested the prediction of the dependence on $\lambda$ of both velocity and diffusion coefficient with direct numerical simulation of the RD equation. The first set of results is shown in Fig. 2. Here data from a simulation of the RD equation with the reaction term (40) are reported and compared with the theoretical prediction (42) for two different values of $\lambda=1$ and $\lambda=5$, in some dimensionless units of the simulation. Notice that the result is indeed sensitive to the

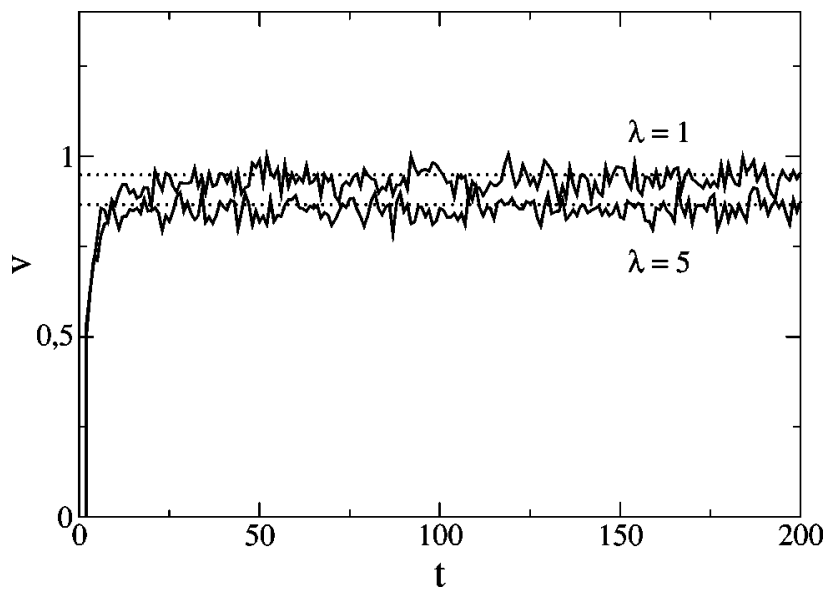

FIG. 2. Change with $\lambda$ of the average renormalized velocity. Values of the parameters are $a=0.1, \varepsilon=0.1, \lambda=1,5$. The theoretical values of the corresponding average velocities from Eq. (42), 0.948 and 0.866 , are also plotted.

microscopic cutoff $\lambda$ of the noise, even though this length is significantly smaller than the front thickness, of the order of 25 , in the same units.

Regarding the diffusion coefficient (43), we measured the mean square displacement of the front position. If we define the front position as

$$
z(t)=\int_{x_{0}}^{\infty} d x \phi(x, t)
$$

then the diffusion coefficient (43) is related to the mean square displacement

$$
\Delta=\sqrt{\left\langle z^{2}\right\rangle-\langle z\rangle^{2}}
$$

as

$$
\Delta^{2}(t) \sim 2 D_{f} t
$$

In Fig. 3 the quantity $\Delta(t)$ is plotted. The values of the parameters are the same as in Fig. 2, while the values of $\lambda$

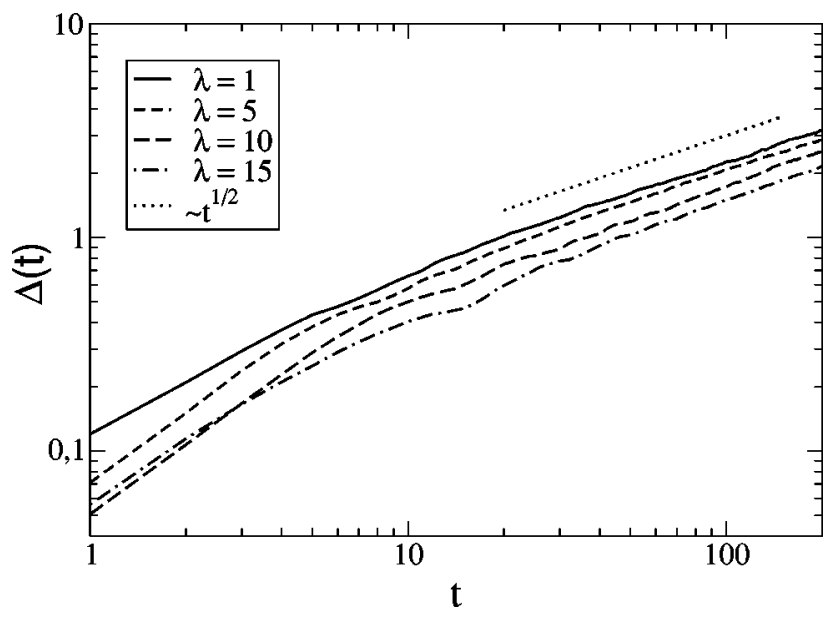

FIG. 3. Change with $\lambda$ of the diffusion coefficient for the front wandering in 1D. Parameters have the same values as in Fig. 2. 
run from 1 to 15 . The diffusive behavior and the dependence on the value of $\lambda$ are manifest.

In this particular case of $1 \mathrm{D}$, a more systematic derivation of the diffusion coefficient has been reported in Ref. [15]. By proper identification of the natural collective variable which describes the front wandering as strictly (not just asymptotically) diffusive, it has been shown that, in fact, the result first found in Ref. [13] is rigorous to first order in $\varepsilon$. Together with the case of equilibrium fluctuations, this is the second rigorous test of our general theory.

\section{B. The $2 \mathrm{D}$ case}

Let us now consider the propagation of a front in the prototype model of Eqs. (39), (40), and (41) in 2D. We have already shown that in two dimensions the eikonal equation reduces to either the EW equation or the KPZ equation, depending on whether the velocity of a planar front is zero or nonzero, respectively. In order to make a numerical check of our theory we consider then the simplest case of zero velocity, with expected EW scaling. This case corresponds to the choice $a=-1 / 2$ in Eq. (40). As seen explicitly in Eq. (42) it turns out that the renormalized velocity is also zero, since the noise does not break the symmetry associated with the double-well form of the deterministic potential. It is important to remark that, unlike the Ginzburg-Landau model discussed in Sec. III for the equilibrium case, the noise is now multiplicative, and no fluctuation-dissipation theorem can be invoked. Hence the first-principles derivation of the fluctuation spectrum in the sharp-interface description is no longer available. We thus rely on the dynamical equation

$\frac{\partial \phi}{\partial t}=D \nabla^{2} \phi+\phi(1-\phi)\left(\phi-\frac{1}{2}\right)+\varepsilon^{1 / 2} \phi(1-\phi) \eta(\mathbf{x}, t)$,

where the noise $\eta$ is defined through the usual correlator of Eq. (2).

Now, our basic goal is to connect this level of description with the eikonal level, determining thereby the noise corrections to this equation. Therefore we assume a stochastic eikonal equation of the form

$$
\begin{gathered}
v_{n}(s)=-\beta \kappa+D_{f}^{1 / 2} \zeta(s, t), \\
\left\langle\zeta(s, t) \zeta\left(s^{\prime}, t^{\prime}\right)\right\rangle=2 \delta\left(s-s^{\prime}\right) \delta\left(t-t^{\prime}\right),
\end{gathered}
$$

where the coefficients $\beta$ and $D_{f}$ are given by

$$
\beta=D, \quad D_{f}=\frac{\int d x u^{0} u_{0} g^{2}\left(\phi_{0}\right)}{\left(u^{0}, u_{0}\right)^{2}} .
$$

Notice that we have directly taken into account that the bare as well as the noise-renormalized velocities are both zero (see, for example, [13]). Also notice that the renormalization of the curvature term is absent for both $\varepsilon$ and $\varepsilon_{\lambda}$ expansions due to the fact that we are now dealing with a front $(N$ $=1$ ).
Now, in order to calculate $D_{f}$, we need to specify the solution of the 1D model. This is known and for $a=-1 / 2$ is given by

$$
\phi_{0}=\frac{1}{2}(1-\tanh k x), \quad k=\frac{1}{2 \sqrt{2}},
$$

and

$$
u^{0}=u_{0}=\frac{d \phi_{0}}{d x} .
$$

The integral in Eq. (50) can then be computed exactly and gives

$$
D_{f}=\varepsilon \frac{9}{35} \sqrt{\frac{2}{1-2 \varepsilon / \lambda^{2}}} .
$$

The above result clearly illustrates the different treatment of the parameters $\varepsilon$ and $\varepsilon / \lambda^{2}$. The result is first order in $\varepsilon$ and contains all orders in $\varepsilon / \lambda^{2}$. It is interesting to remark that the partial resummation of all orders in $\epsilon$ captures important nonperturbative phenomena. For instance, the divergence of $D_{f}$ at $\varepsilon / \lambda^{2}=1 / 2$ reflects the fact that at this point the front itself is destroyed, or, equivalently, the front thickness becomes infinite. This is equivalent to reaching a critical point, except that this is not the equilibrium one because the noise is multiplicative. Remarkably, our result for the additive noise case does not capture that feature because it is only first order in the noise strength. In the case with an asymmetric double-well potential, which has a finite front velocity, it was explicitly checked numerically in Ref. [13] that the diffusion coefficient of the front has a nonmonotonic dependence on $\varepsilon / \lambda^{2}$. Most importantly, it vanishes at a finite value of $\varepsilon / \lambda^{2}$ that corresponds exactly to the point where the front reaches the pushed-pulled transition. Again we see that the resummation of orders $\varepsilon / \lambda^{2}$ captures important physical information (see the discussion in Sec. V).

We now come back to the numerical test of the EW scaling of our particular symmetric case, with the identification (28). Accordingly, we can rewrite the complete power spectrum as it is known theoretically [34] in terms of the coefficients that we just calculated, and compare it with data from a direct simulation of the field model Eq. (48).

From [34] the power spectrum $S$ reads

$$
S(q, t)=\frac{1}{L} \frac{D_{\mathrm{EW}}}{\nu q^{2}}\left[1-\exp \left(-2 \nu q^{2} t\right)\right] .
$$

In terms of the parameters in the original field equation this yields

$$
S(q, t)=\frac{1}{L} \frac{D_{f}}{D q^{2}}\left[1-\exp \left(-2 D q^{2} t\right)\right]
$$

where $D_{f}$ is given by Eq. (53). Accordingly, the fluctuations of the front position in the RD model Eq. (39) for length scales larger than the front thickness itself must obey the 


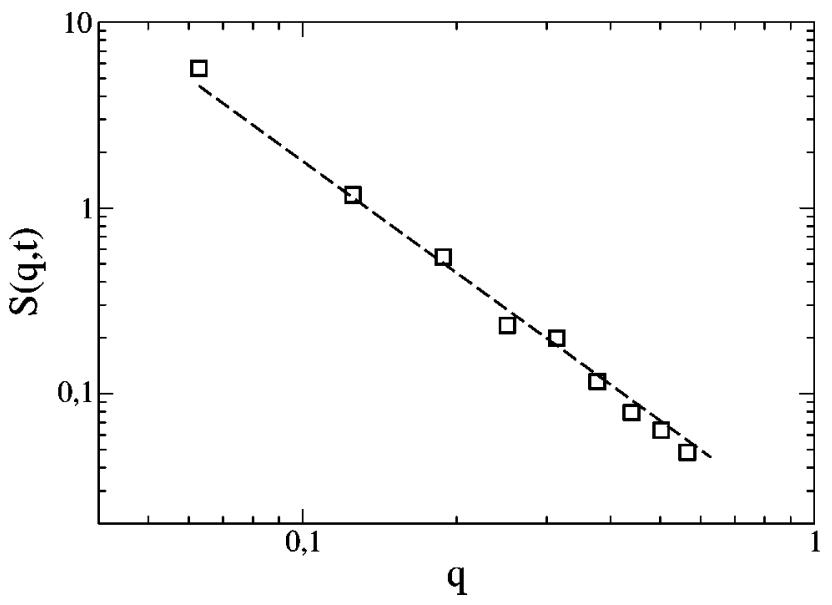

FIG. 4. Numerical data from $2 \mathrm{D}$ simulation of the RD field equations for the prototype model with $a=-1 / 2$ and analytical prediction for the power spectrum (55) in the scaling region. The time here is 1000 , and the parameters of the simulation are $L$ $=100, \varepsilon=5, \lambda=5$.

spectrum defined by Eq. (55). It is worth remarking that the prediction is not only for the universal features, namely, the shape of the scaling function and the exponents, but for the actual absolute values of the spectrum. We have performed simulations of the field RD model with a correlator of the form

$$
\begin{aligned}
\left\langle\eta(\mathbf{x}, t) \eta\left(\mathbf{x}^{\prime}, t^{\prime}\right)\right\rangle= & \frac{1}{\lambda^{2}}\left(1-\left|x-x^{\prime}\right| / \lambda\right)\left(1-\left|\vec{y}-\vec{y}^{\prime}\right| / \lambda\right) \\
& \times \Theta\left(1-\left|x-x^{\prime}\right| / \lambda\right) \\
& \times \Theta\left(1-\left|y-y^{\prime}\right| / \lambda\right) \delta\left(t-t^{\prime}\right)
\end{aligned}
$$

This corresponds to assuming that at any time the noise takes the same value in a square of side $\lambda$, uncorrelated with the neighboring squares. This is done for simplicity but no significant dependence is expected on the details of the shape of the spatial correlation provided $\lambda$ is kept smaller than the front thickness.

We have studied the fluctuations of the internal level curve of the front $\phi=1 / 2$. In Fig. 4 we show the scaling region, with the correct slope and location of the curve. More remarkably, Fig. 5 shows the measured spectra for the simulation of the field equations compared to the prediction given by Eq. (55). It should be stressed that in this comparison there is no free parameter. It is also interesting to observe the deviations from the prediction at length scales smaller than the front thickness. In this high- $q$ region the data also collapse but not to EW scaling. An estimate of the exponent $\alpha$ in this region is around $3 / 2$.

For values of $a$ such that the front has a finite velocity, one would expect that the scaling would be given by that of the KPZ universality class. Although the scaling function in that case is not exactly known, the prediction of our eikonal equation is expected to fit the data for the corresponding RD model also without free parameters. We have not checked this case because it is obviously more involved and less con-

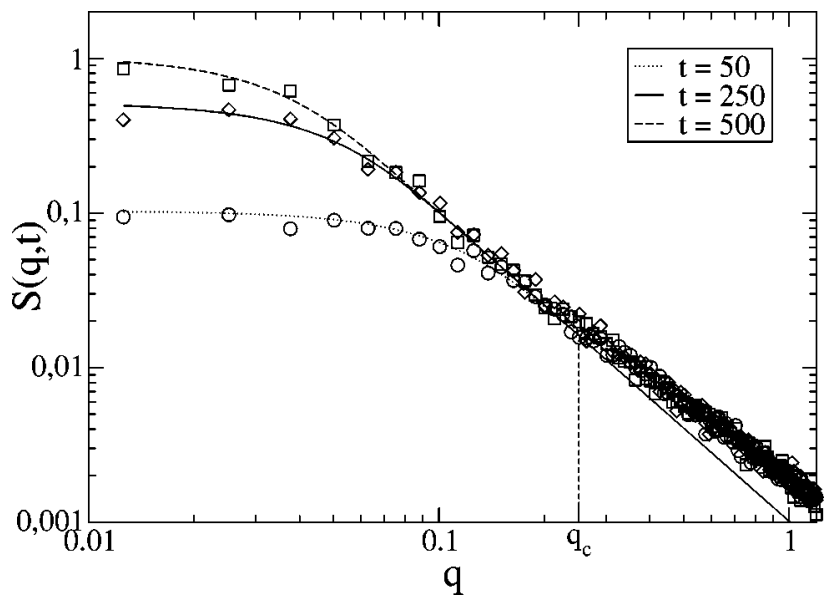

FIG. 5. Numerical data from 2D simulation of the RD field equations for the prototype model with $a=-1 / 2$ and analytical prediction for the power spectrum (55). The three sets of data points refer to times 50,250, and 500. The parameters of the simulation are $L=500, \varepsilon=1, \lambda=2$. The value of $q_{c}$ corresponds to a wavelength of the order of the front thickness. The analytical prediction is intended only for $q<q_{c}$.

clusive given the practical difficulties in already reaching the scaling regime at the eikonal level of description [46].

From a practical point of view it is to be remarked that the noise intensity cannot be increased arbitrarily in a simulation without destroying the front itself. This can be easily seen in a numerical simulation. Although the noise vanishes asymptotically in the stationary states $\phi=0$ and $\phi=1$, if noise is sufficiently strong it may be capable of nucleating the other state in the region not too far from the front. We have found that this effect is more pronounced in the region behind the front $\phi=1$. For a given $\lambda$ there will typically be a maximum value of $\varepsilon$ after which the front is essentially destroyed. If we increase $\lambda$ the effect is milder. In order to make the front roughening appreciable in not too large system sizes, it is thus convenient to have a moderately large $\lambda$, which in turn will allow larger values of $\varepsilon$. Typical values that we have considered are in the range of $\lambda=2,5$ in units for which the front thickness is of the order of 25 .

\section{THE PUSHED-PULLED TRANSITION}

As we have mentioned, two classes of fronts must be distinguished from a dynamical point of view, the so-called pushed and pulled fronts $[4,29]$. The simplest is the pushed case, in which the front propagation depends on the full nonlinear structure of the equation of motion and the front is said to be "pushed" by its internal part. This is usually the case when the invaded state is locally stable. On the contrary, if the invaded state is unstable, it can happen that the relevant dynamics takes place in the semi-infinite leading edge region ahead of the front itself. Then the propagation of the front is governed by the growth and spreading of linear perturbations in that region, which "pull" the front. In this case the linearization about the unstable state accounts for its dynamical behavior [4], but there is degeneracy of propagating veloci- 


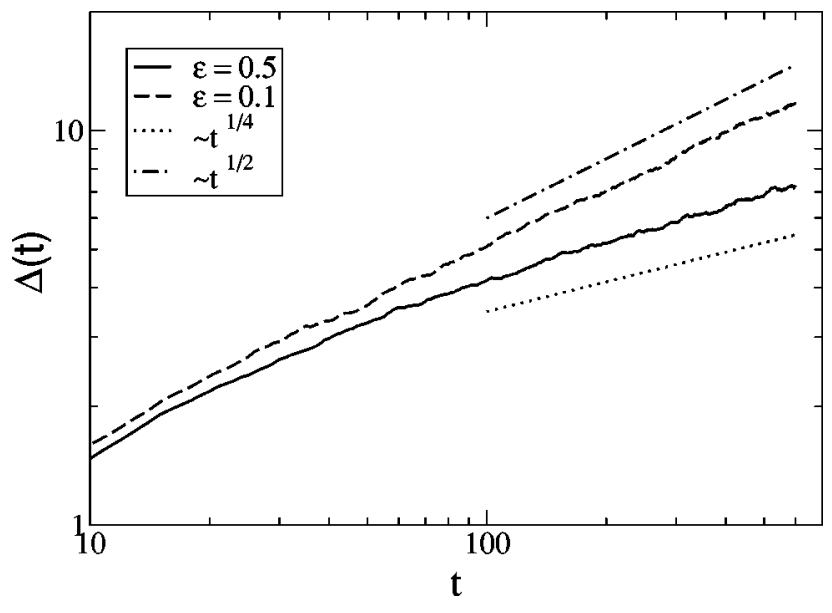

FIG. 6. The pushed-pulled transition in 1D. The system size is $L=2000$ and averages have been carried out on 3600 realizations of noise in the pulled case and about 1000 in the pushed one.

ties [2]. In the present context, the most important distinction between the two situations is that, while for pushed fronts the relaxation of bulk modes is exponential, for pulled fronts it is algebraic, as a result of the fact that the linearized operator describing perturbations around the stationary propagating mode is gapless. This means that for pulled fronts there is no natural time-scale separation which allows for the decoupling of the interface modes from the bulk ones. Our derivation, and the whole idea of a kinematic moving-boundary approximation, is not valid for pulled fronts [29]. Nevertheless, we will show that our theory does correctly predict its failure at the pushed-pulled transition.

The intrinsic differences between pushed and pulled fronts also show dramatically in the statistics of fluctuations. In $1 \mathrm{D}$, for instance, the front wandering turns from diffusive (pushed fronts) to subdiffusive (pulled fronts) [13,15]. In turn, pulled fronts in 2D with multiplicative noise have been found to belong to a different universality class from the ordinary KPZ one [16,17], as opposed to the KPZ scaling of pushed fronts.

In Ref. [13] it was already observed that the diffusion coefficient of fronts in 1D was a nonmonotonic function of $\varepsilon / \lambda$ which vanished at a finite value of that parameter. After that point, subdiffusive behavior was found. The picture was confirmed and completed in Ref. [15]. Here we want to stress that the point where that transition occurs corresponds $e x$ actly to the pushed-pulled transition. In fact, the renormalized equation defined by Eq. (5) for the prototype model takes the same form as the original one, with renormalized coefficients. The deterministic equation has the transition to the pulled regime at $a=1 / 2$. The same transition in the renormalized equation occurs at $\tilde{a}=1 / 2$ where $\tilde{a}=a+\varepsilon_{\lambda}$. It is thus clear that, for parameter values of the deterministic equation in the pushed regime, increasing noise intensity $\varepsilon$ or decreasing $\lambda$ will imply a transition to the pulled regime. While the front velocity will not experience any dramatic effect, the fluctuations (wandering in 1D or roughening in 2D) will be dramatically affected, since the universality class will change. In Fig. 6 we show the change from diffusive to subdiffusive behavior induced solely by a change in the ef- fective noise intensity $\varepsilon_{\lambda}$. Once more the dynamical importance of the noise correlation length $\lambda$ in the long wavelength behavior of the front is remarkable. The same effect should be expected in 2D, namely, the scaling could be KPZ or non-KPZ depending solely on noise parameters. To our knowledge, this is the first time that such a dramatic effect of noise has been reported. Remarkably enough, while our eikonal description is not able to describe the second regime, it does predict the transition at the right values of the parameters.

\section{DISCUSSION AND CONCLUSIONS}

The formulation in terms of kinematic eikonal-like equations provides a useful framework for studying front or pulse propagation when one is interested in long spatial and temporal scales. This kind of equation has advantages both for numerical simulations and for theoretical analysis, and has fruitfully been used, for instance, in the phenomenological modeling of excitable wave propagation in disordered and noisy media, by the ad hoc procedure of adding fluctuations to a generic eikonal equation. In this paper we have derived stochastic eikonal equations from the more microscopic RD field equations with noise, which can be multiplicative.

The derivation presented here relies on the hypothesis of separation of scales between the front dynamics at large scales and the internal degrees of freedom of the front. This means that it is not valid for pulled fronts, which are indeed known not to have a local, eikonal-like description even in the absence of noise. However, even for the pushed case, the usual projection techniques for derivation of sharp-interface equations cannot be simply extended to the stochastic case due to the fact that the noise contains the full range of length and time scales. Hence our derivation is formulated within a coarse-graining scheme, which we claim makes sense for the kind of problems that admit a local, eikonal-like equation. Derivation of sharp-interface approximations with noise has been possible so far for thermal noise only, in systems with local equilibrium. For multiplicative, generically external noise, however, the absence of a free energy and a fluctuation-dissipation theorem require an alternative scheme based on dynamical equations. We have explicitly checked that our scheme is exact for the cases of equilibrium fluctuations and also reproduces a rigorous result for multiplicative noise in 1D. For the general case, however, we rely on numerical tests to fully justify its validity. In any case, the excellent agreement with numerical and analytical tests clearly suggests that a more rigorous derivation should be possible. An extension of our procedure is also conceivable in RD systems for which a nonlocal sharp-interface description exists, for instance, in solidification or viscous fingering.

One of the main points of this paper has been to clarify the role of the spatial cutoff of noise correlations $\lambda$. On the basis of recent rigorous mathematical findings on the spatiotemporal white noise limit, we have argued and shown in explicit examples that the common splitting of the Stratonovich white noise into a term which is singular as $\lambda \rightarrow 0$ plus an Itô noise does capture the correct dependence on $\lambda$ of macroscopic quantities (at scales much larger than $\lambda$ ). The re- 
maining spatially correlated Itô noise will carry only a weak dependence on $\lambda$, noticeable at length scales of the order of $\lambda$, and can be treated perturbatively. The $\lambda \rightarrow 0$ limit can thus be taken safely for the remaining Itô noise, once the singular part has been extracted. Within this scheme, macroscopic quantities such as the front velocity or front roughening properties depend separately on the noise strength $\varepsilon$ and on $\varepsilon / \lambda^{d}$. Incidentally, this implies the possibility of measuring the (microscopic) noise parameters $\varepsilon$ and $\lambda$ from the macroscopic dynamics of the fronts. Most remarkably, a partial resummation scheme can be naturally defined for the parameter $\varepsilon / \lambda^{d}$ which captures some important, nonperturbative physical phenomena that would be missed otherwise, such as the transition from pushed to pulled regimes, or the destruction of the front associated with a divergence of the front thickness. These phenomena, at the edge of validity of the sharp-interface approximation, are detected, respectively, by the vanishing and the divergence of the effective noise strength in the eikonal equation.

Eikonal stochastic equations like the ones derived here are directly related to the EW and KPZ equations of kinetic roughening. By using known results for these equations and the results presented here one can predict roughening properties of noisy pulses or fronts appearing in field equations. We have used this correspondence to check the predictions in the case of zero velocity and additive noise, for which the general theory of equilibrium fluctuations can be directly applied to the RD equation. Analytical results obtained for the corresponding (Edwards-Wilkinson) eikonal equation are identical (including prefactors) to that independent calculation for the RD system.

We have also applied our results to a prototype model with multiplicative noise constructed to represent a variety of different front propagation regimes by changing a single control parameter. While this model has already been used to study the effects of noise on 1D fronts, we have addressed here a more specific $2 \mathrm{D}$ effect, the case of front roughening. In the zero-velocity case, simulations of the reactiondiffusion equation presented very good agreement with the predictions of roughening for the corresponding EW equation with no adjustable parameter. The results show that the dependence on $\lambda$ is quantitatively important even if $\lambda$ is significantly smaller than the front thickness, which may seem counterintuitive. Although we have not checked the analogous results for a nonzero velocity, which would correspond to the KPZ equation, we expect our results to be valid also in this case.

Most interestingly we have explicitly checked the prediction of qualitative changes as $\varepsilon / \lambda^{d}$ is varied, such as the transition from pushed to pulled propagation regimes. We have directly observed the change in the wandering exponent in $1 \mathrm{D}$ as $\lambda$ is decreased. The immediate extension of this result implies that a transition from KPZ to non-KPZ scaling is to be expected in higher dimensions. We thus conclude that the dependence on the spatial cutoff of the noise may have dramatic effects, not only on the nonuniversal quantities but also on the universal ones.

In summary, we have derived stochastic eikonal equations from stochastic RD equations completely specifying the pa- rameters of the noise. Although this is not a systematic derivation, we have presented a wealth of independent evidence of the validity of our results both from analytical calculations and from numerical simulations of different systems and looking at distinct noise effects, both qualitative and quantitative. These results should be of interest for theoretical and practical purposes both in the study of kinetic roughening and in the context of propagation of chemical waves.

\section{ACKNOWLEDGMENTS}

This work originated in earlier collaboration with J. M. Sancho and F. Sagués, and has also benefited from later discussions with W. van Saarloos. We acknowledge financial support from Dirección General de Investigación Científica y Técnica (Spain) (Projects No. BFM2000-0624-C03-02 and No. BXX2000-0638-C02-02), Comissionat per a Universitats i Recerca (Spain) (Projects No. 1999SGR00145 and No. 2000XT-0005), and the European Commission (Project No. TMR-ERBFMRXCT96-0085). We also acknowledge computing support from Fundació Catalana per a la RecercaCentre de Supercomputació de Catalunya (Spain).

\section{APPENDIX: SYSTEMATIC EXPANSION}

In some circumstances, the eigenvalue problem defined by Eq. (10) may not be solvable analytically, due to the functional form of $\mathbf{h}\left(\boldsymbol{\phi}_{0}\right)$, which has been renormalized by noise, even though the "bare" problem may be solvable. In such cases it may be useful to solve for the case $\varepsilon=0$ (no noise) and obtain corrections in a systematic expansion in $\varepsilon$. This is the aim of this appendix.

Let us consider then the zero-noise eigenvalue problem defined by the (deterministic) 1D equation

$$
0=\hat{D} \frac{\partial^{2} \boldsymbol{\phi}_{\mathcal{D}}}{\partial r^{2}}+v_{0} \frac{\partial \boldsymbol{\phi}_{\mathcal{D}}}{\partial r}+\mathbf{f}\left(\boldsymbol{\phi}_{\mathcal{D}}\right)
$$

We now expand the fields of the noisy 2D problem of Eq. (9) as perturbations in $\varepsilon$ and $\kappa$ of the solution of Eq. (A1):

$$
\begin{gathered}
\boldsymbol{\phi}(r, s, t)=\boldsymbol{\phi}_{\mathcal{D}}(r)+\delta \boldsymbol{\phi}(r, s, t), \\
v_{n}(s, t)=v_{0}+\alpha \varepsilon+\beta \kappa(s, t)+\delta v(s, t) .
\end{gathered}
$$

We get at the linear order

$$
\begin{aligned}
0= & \hat{\Gamma}_{0} \delta \boldsymbol{\phi}+\varepsilon \mathbf{G}\left(\boldsymbol{\phi}_{\mathcal{D}}\right)+(\alpha \varepsilon+\beta k+\delta v) \frac{\partial \boldsymbol{\phi}_{\mathcal{D}}}{\partial r} \\
& +\hat{D} k \frac{\partial \boldsymbol{\phi}_{\mathcal{D}}}{\partial r}+\varepsilon^{1 / 2} \boldsymbol{\Omega}\left(\boldsymbol{\phi}_{\mathcal{D}} ; r, s ; t\right),
\end{aligned}
$$

where

$$
\hat{\Gamma}_{0}=\hat{D} \frac{\partial^{2}}{\partial r^{2}}+v_{0} \frac{\partial}{\partial r}+\left.\frac{\partial \mathbf{f}}{\partial \boldsymbol{\phi}}\right|_{\boldsymbol{\phi}=\boldsymbol{\phi}_{\mathcal{D}}} .
$$

Now the right eigenvector of $\hat{\Gamma}_{0}$ reads 


$$
\mathbf{u}_{(0)}=\frac{\partial \boldsymbol{\phi}_{\mathcal{D}}}{\partial r}
$$

which is independent of both $\varepsilon$ and $k$. Taking Eqs. (A4) and performing the scalar product with the left eigenvector $\mathbf{u}^{(0)}$, we get

$$
\begin{aligned}
& k\left(\mathbf{u}^{(0)}, \hat{D} \mathbf{u}_{(0)}\right)+(\alpha \varepsilon+\beta k)\left(\mathbf{u}^{(0)}, \mathbf{u}_{(0)}\right) \\
& +\varepsilon\left(\mathbf{u}^{(0)}, \mathbf{G}\left(\boldsymbol{\phi}_{\mathcal{D}}\right)\right)+\varepsilon^{1 / 2}\left(\mathbf{u}^{(0)}, \boldsymbol{\Omega}\left(\boldsymbol{\phi}_{\mathcal{D}} ; r, s ; t\right)\right) \\
& +\delta v(s, t)\left(\mathbf{u}^{(0)}, \mathbf{u}_{(0)}\right)=0 .
\end{aligned}
$$

As curvature and fluctuations are linear perturbations we get

$$
\alpha=-\frac{\left(\mathbf{u}^{(0)}, \mathbf{G}\left(\boldsymbol{\phi}_{\mathcal{D}}\right)\right)}{\left(\mathbf{u}^{(0)}, \mathbf{u}_{(0)}\right)}, \quad \beta=-\frac{\left(\mathbf{u}^{(0)}, \hat{D} \mathbf{u}_{(0)}\right)}{\left(\mathbf{u}^{(0)}, \mathbf{u}_{(0)}\right)},
$$

and

$$
\delta v(s, t)=-\varepsilon^{1 / 2} \frac{\left(\mathbf{u}^{(0)}, \boldsymbol{\Omega}\left(\boldsymbol{\phi}_{\mathcal{D}} ; r, s ; t\right)\right)}{\left(\mathbf{u}^{(0)}, \mathbf{u}_{(0)}\right)} .
$$

The stochastic eikonal equation is now

$$
v_{n}(s, t)=v_{0}+\alpha \varepsilon+\beta \kappa(s, t)+D_{f}^{1 / 2}(\varepsilon) \zeta(s, t),
$$

where the noise $\zeta(s, t)$ is a zero-mean Gaussian white process with

$$
\left\langle\zeta(s, t) \zeta\left(s^{\prime}, t^{\prime}\right)\right\rangle=2 \delta\left(s-s^{\prime}\right) \delta\left(t-t^{\prime}\right)
$$

and

$$
D_{f}(\varepsilon)=\varepsilon \frac{\int d r \sum_{i, j} u_{i}^{(0)} u_{j(0)} g_{i}\left(\boldsymbol{\phi}_{\mathcal{D}}\right) g_{j}\left(\boldsymbol{\phi}_{\mathcal{D}}\right)}{\left(\mathbf{u}^{(0)}, \mathbf{u}_{(0)}\right)^{2}} .
$$

[1] C. Cross and P. C. Hohenberg, Rev. Mod. Phys. 65, 851 (1993).

[2] W. van Saarloos, Phys. Rev. Lett. 58, 2571 (1987); Phys. Rev. A 37, 211 (1988); 39, 6367 (1989).

[3] P. Collet and J. P. Eckmann, Instabilities and Fronts in Extended Systems (Princeton University Press, Princeton, NJ, 1990).

[4] U. Ebert and W. van Saarloos, Physica D 146, 1 (2000).

[5] Chemical Waves and Patterns, edited by R. Kapral and K. Showalter (Kluwer Academic, Dordrecht, 2001).

[6] A. S. Mikhailov, Foundations of Synergetics I (SpringerVerlag, Berlin, 1995).

[7] J. J. Tyson and J. P. Keener, Physica D 32, 327 (1988).

[8] E. Meron, Phys. Rep. 218, 1 (1992).

[9] A. S. Mikhailov, V. A. Davydov, and V. S. Zykov, Physica D 70, 1 (1994).

[10] J. M. Davidenko, A. M. Pertsov, R. Salomonz, W. Baxter, and J. Jalife, Nature (London) 335, 349 (1992); A. M. Pertsov, J. M. Davidenko, R. Salomonz, W. Baxter, and J. Jalife, Circ. Res. 72, 631 (1992); A. Karma, Proc. Natl. Acad. Sci. U.S.A. 97, 5687 (2000).

[11] J. Riordan, C. R. Doering, and D. ben-Avraham, Phys. Rev. Lett. 75, 565 (1995).

[12] J. Armero, J. M. Sancho, J. Casademunt, A. M. Lacasta, L. Ramírez-Piscina, and F. Sagues, Phys. Rev. Lett. 76, 3045 (1996).

[13] J. Armero, J. Casademunt, L. Ramírez-Piscina, and J. M. Sancho, Phys. Rev. E 58, 5494 (1998).

[14] D. A. Kessler and H. Levine, Nature (London) 394, 556 (1998).

[15] A. Rocco, U. Ebert, and W. van Saarloos, Phys. Rev. E 62, R13 (2000).

[16] G. Tripathy and W. van Saarloos, Phys. Rev. Lett. 85, 3556 (2000).

[17] G. Tripathy, A. Rocco, J. Casademunt, and W. van Saarloos, Phys. Rev. Lett. 86, 5215 (2001).

[18] A. Rocco, J. Casademunt, U. Ebert, and W. van Saarloos,
Phys. Rev. E 65, 012102 (2002).

[19] A. Torcini, A. Vulpiani, and A. Rocco, Eur. Phys. J. B 25, 333 (2002).

[20] I. Sendiña-Nadal, M. Gómez-Gesteira, V. Pérez-Munuzuri, V. Pérez-Villar, J. Armero, L. Ramírez-Piscina, J. Casademunt, J. M. Sancho, and F. Sagués, Phys. Rev. E 56, 6298 (1997).

[21] I. Sendiña-Nadal, A. P. Pérez-Munuzuri, D. Vives, V. PérezMunuzuri, J. Casademunt, L. Ramírez-Piscina, J. M. Sancho, and F. Sagués, Phys. Rev. Lett. 80, 5437 (1998).

[22] I. Sendiña-Nadal, S. Alonso, V. Pérez-Muñuzuri, M. GómezGesteira, V. Pérez-Villar, L. Ramírez-Piscina, J. Casademunt, J. M. Sancho, and F. Sagués, Phys. Rev. Lett. 84, 2734 (2000).

[23] S. Kàdàr, J. Wang, and K. Showalter, Nature (London) 391, 770 (1998).

[24] E. Brunet and B. Derrida, Phys. Rev. E 56, 2597 (1997).

[25] D. A. Kessler, Z. Ner, and L. M. Sander, Phys. Rev. E 58, 107 (1998).

[26] D. Panja and W. van Saarloos, e-print cond-mat/0110344.

[27] E. Moro, Phys. Rev. Lett. 87, 238303 (2001).

[28] V. Hakim and A. Karma, Phys. Rev. E 60, 5073 (1999).

[29] U. Ebert and W. van Saarloos, Phys. Rep. 337, 139 (2000).

[30] S.-L. Wang, R. F. Sekerka, A. A. Wheeler, B. T. Murray, S. R. Coriell, R. J. Braun, and G. B. McFadden, Physica D 69, 189 (1993).

[31] A. Karma and W.-J. Rappel, Phys. Rev. E 53, R3017 (1996).

[32] R. Folch, J. Casademunt, A. Hernández-Machado, and L. Ramírez-Piscina, Phys. Rev. E 60, 1724 (1999); 60, 1734 (1999).

[33] A.-L. Barabási and H. E. Stanley, Fractal Concepts in Surface Growth (Cambridge University Press, Cambridge, England, 1995).

[34] J. Krug, Adv. Phys. 46, 139 (1997).

[35] M. Kardar, G. Parisi, and Y. C. Zhang, Phys. Rev. Lett. 56, 889 (1986).

[36] P. C. Hohenberg and B. I. Halperin, Rev. Mod. Phys. 49, 435 (1977). 
[37] S. A. Safran, Statistical Thermodynamics of Surfaces, Interfaces, and Membranes (Addison-Wesley, Reading, MA, 1994).

[38] A. Karma, Phys. Rev. Lett. 70, 3439 (1993).

[39] A. Karma and W.-J. Rappel, Phys. Rev. E 60, 3614 (1999).

[40] R. González-Cinca, L. Ramírez-Piscina, J. Casademunt, and A. Hernández-Machado, Phys. Rev. E 63, 051602 (2001).

[41] C. W. Gardiner, Handbook of Stochastic Methods for Physics, Chemistry and the Natural Sciences (Springer-Verlag, Berlin, 1983); N. G. van Kampen, Stochastic Processes in Physics and
Chemistry (North-Holland, Amsterdam, 1981).

[42] E. A. Novikov, Zh. Éksp. Teor. Fiz. 47, 1919 (1964) [Sov. Phys. JETP 20, 1290 (1965)].

[43] S. Gyöngy, Stochastic Proc. Appl. 73, 271 (1998).

[44] E. Alòs, J. A. León, and D. Nualart, Prob. Theory Relat. Fields 115, 41 (1999).

[45] S. F. Edwards and D. R. Wilkinson, Proc. R. Soc. London, Ser. A 381, 177 (1982).

[46] M. Beccaria and G. Curci, Phys. Rev. E 50, 4560 (1994). 